\title{
The influence of direct, delta, and modulation QD Si doping on InAs/GaAs quantum dot solar cells
}

\author{
Dongyoung Kim, Shun Chan, Mingchu Tang, Jiang Wu, and Huiyun Liu \\ Department of Electronic and Electrical Engineering, University College London, \\ Torrington Place, London WC1E 7JE, United Kingdom
}

\begin{abstract}
The effects of direct, delta, and modulation Si QD doping on InAs/GaAs QDSCs are studied. The PL, EQE, and J-V characterisation results show a clear relationship between the doping methods and the non-radiative recombination. All doped QDSCs exhibited increase in the Voc due to reduced thermal coupling from QD Si doping. Delta and modulation-doped QDSCs exhibit further improvements in Voc due to reduced nonradiative recombination. Moreover, the modulation-doped QDSC shows improvements in both the current density and the voltage compared with the directly doped QDSC.

Index Terms - Quantum dots, intermediate band, Si doping, molecular beam epitaxy.
\end{abstract}

\section{INTRODUCTION}

One of the main challenges in implementing high-efficiency QD IBSCs is to prevent the decrease in $\mathrm{V}_{\mathrm{OC}}$ when QDs are introduced to the structure. The $\mathrm{V}_{\mathrm{OC}}$ drop originates from the thermal coupling between IB and conduction band (CB) that occurs when carriers can be thermally excited and relaxed between IB and CB, and hence, there is no need for twophoton excitation. Studies have suggested possible solutions to prevent the $\mathrm{V}_{\mathrm{OC}}$ drop [1]-[3]. Amongst those, Okada et al. demonstrated the two-photon absorption via intermediate quantum states in InAs/GaNAs QD IBSC at room temperature by direct Si-doping of QDs [1]. However, to date, there has been no published study comparing IB QDSCs with different types of QD Si doping.

In this project, three QDSCs with different doping methods, alongside two reference cells, have been grown by MBE and characterised by photoluminescence (PL), external quantum efficiency (EQE), and $\mathrm{J}-\mathrm{V}$ measurements. Three doping methods investigated in this work are direct doping, delta doping, and modulation doping. Firstly, Si direct doping is a doping method that creates a built-in field at the interface between the QDs and wetting layer (WL) by direct Si doing in QDs. The built-in energy formed at the QDs/WL interface increases the thermal activation energy, which leads to a reduced thermal activity from IB to CB [4]. It is believed that Si direct doping can help achieve voltage recovery without employing higher bandgap material. Secondly, it is believed that Si delta doping embedded into intrinsic spacer the QDs can improve sub bandgap collection efficiency [5]. With deltadoped QDs, the confined states between $\mathrm{CB}$ and VB are already partially filled with carriers. Therefore, it is possible for photons with sub bandgap energy to help carriers transit into $\mathrm{CB}$ continuum. Sablon et al. have demonstrated an increased sub bandgap current collection after the introduction of delta doing [6], whereas others observed carrier extraction through two-photon sequential absorption [1], [7]. Also, Morioka et al. have shown that band flattened by delta-doped QDs significantly reduces Shockley-Read-Hall (SRH) recombination rate and dark current [8]. Lastly, Si modulation doping is a doping method where doping is introduced in a thin spacer layer between QD layers [9].

\section{EXPERIMENTAL WORK}

The GaAs reference SC sample was grown by a solid-source molecular beam epitaxy (MBE) on $\mathrm{n}^{+}$GaAs (100) substrates. The GaAs reference SCs was grown with the same p-i-n structure that consists of a $200 \mathrm{~nm} \mathrm{GaAs}$ buffer layer with $\mathrm{Si}$ doping density of $1 \times 10^{18} \mathrm{~cm}^{-3}, 30 \mathrm{~nm} \mathrm{Al}_{0.33} \mathrm{Ga}_{0.67} \mathrm{As}$ back surface field (BSF) with Si doping density of $1 \times 10^{18} \mathrm{~cm}^{-3}$, $420 \mathrm{~nm}$ intrinsic region GaAs, $1000 \mathrm{~nm}$ GaAs base with $\mathrm{Si}$ doping density of $1 \times 10^{17} \mathrm{~cm}^{-3}, 250 \mathrm{~nm} \mathrm{GaAs}$ emitter with Be doping density of $2 \times 10^{18} \mathrm{~cm}^{-3}, 30 \mathrm{~nm} \mathrm{Al}{ }_{0.6} \mathrm{Ga}_{0.4} \mathrm{As}$ window layer with Be doping density of $2 \times 10^{18} \mathrm{~cm}^{-3}$, and $50 \mathrm{~nm}$ GaAs contact layer with Be doping density of $1 \times 10^{19} \mathrm{~cm}^{-3}$.

The reference QDSC sample has an identical structure to the reference GaAs SC except for its intrinsic region. The intrinsic region of the undoped QDSC consists of 20 stacks of 2.1 monolayer (ML) InAs separated by a $20 \mathrm{~nm}$ GaAs spacer. The QDs were grown by the Stranski-Krastanov mode at substrate temperature of $\sim 500{ }^{\circ} \mathrm{C}$. High-growth-temperature $\mathrm{GaAs}$ spacer layers were applied during the growth of QDs to suppress the formation of dislocations [10]-[12].

The same QDSC structure as the undoped reference QDSC was used for the doped QDSCs except for the active regions. For the directly doped QDSC, Si-dopants were directly applied to the QDs during the QD growths [4], and the QD layers were capped with $20 \mathrm{~nm}$ undoped GaAs interlayer. For the delta-doped QDSC, undoped QD layers were capped with a $4 \mathrm{~nm}$ undoped GaAs interlayer, before the deposition of a thin Si layer which was then capped with a $16 \mathrm{~nm}$ undoped GaAs interlayer. The modulation-doped QDSC structure has undoped QD layers capped with a $7 \mathrm{~nm}$ undoped GaAs interlayer. The Si doping is then introduced to the following 6 $\mathrm{nm}$ GaAs interlayer which is then capped with a $7 \mathrm{~nm}$ undoped 
GaAs interlayer. For all doped QDSCs, the QD Si doping density remained at the same level (14 electrons per QD).

For device fabrication, Au-Zn alloy (95\% Au, $5 \% \mathrm{Zn})$ was thermally evaporated to form a ( $200 \mathrm{~nm}$ thick) grid-pattern p-type electrode using a metal shadow mask. For the n-type electrode, $10 \mathrm{~nm} \mathrm{Ni}, 100 \mathrm{~nm}$ Au-Ge ( $88 \% \mathrm{Au}, 12 \% \mathrm{Ge}), 30$ $\mathrm{nm} \mathrm{Ni}$, and $200 \mathrm{~nm} \mathrm{Au}$ were thermally evaporated onto the entire back surface, and thermally annealed at $400{ }^{\circ} \mathrm{C}$ for $60 \mathrm{~s}$. No anti-reflective coating or surface passivation was applied to these SCs.

For device characterization, A Veeco Nanoscope V atomic force microscope (AFM) was used to characterise the morphology of an uncapped QD layer. Temperature-dependent and power-dependent photoluminescence (PL) measurements were performed using a diode-pumped solid-state laser. Current density vs voltage $(\mathrm{J}-\mathrm{V})$ characteristics were obtained by using a solar simulator with a xenon lamp under one-sun air mass (AM) $1.5 \mathrm{G}$ illumination. Photocurrent measurements were performed with a halogen lamp chopped to a frequency of $188 \mathrm{~Hz}$ through a monochromator. The monochromatic beam was calibrated with a silicon photo-diode and the data was analysed to obtain the external quantum efficiency (EQE) at room temperature.

\section{RESULTS AND DISCUSSION}

Figure 1 and Figure 2 show the PL spectra and the integrated PL intensities for all QDSC samples at RT $(300 \mathrm{~K})$. All samples display two peaks at $\sim 950 \mathrm{~nm}$ and $\sim 1100 \mathrm{~nm}$ which correspond to the emissions from the WL and the QD ground state, respectively. Improved PL intensity is observed for the directly doped QDSC sample compared with the undoped QDSC sample. This enhanced PL emission suggest that PL quenching is suppressed due to an increased thermal activation energy. The increase in the thermal activation energy is attributed to the potential barrier between GaAs and the QDs formed by Si doping, as demonstrated by Lam et al. [4]. This potential barrier can suppress the thermal escape of electrons from QDs, and hence contribute to the recovery of $\mathrm{V}_{\mathrm{OC}}$. A further increase in the PL intensity are observed for the deltadoped QDSC. When Si doping is introduced within QDs, the presence of the Si atoms can lead to the destruction of the crystal lattice, and hence introduce non-radiative recombination centres [14]. However, it has been reported that placing dopants away from the QDs can reduce the number of point defects in the active QD region [15]. The increase in the PL emissions of the delta-doped QDSC samples suggests that delta doping accompanies less crystal lattice destruction as the $\mathrm{Si}$ dopants are introduced outside the QDs, which means smaller number of non-radiative recombination centres in presence. The highest integrated PL intensity is observed for the modulation-doped QDSC. This could be because of the presence of the $7 \mathrm{~nm}$ GaAs spacer layer between the Si-doped layer and the QD layer. In other words, the introduction the $\mathrm{Si}$ dopants could have a less significant influence on the morphology of the QDs during the growth as the QDs are separated from the Si-doped layer.

Figure 3 illustrates the EQE spectra of all SC samples. It shows that all SCs with Si doping (direct, delta, and modulation) have lower spectral response in the GaAs region $(400 \mathrm{~nm}-900 \mathrm{~nm})$. This can be attributed to the decrease in depletion region after Si doping, which reduces the effective absorption area. For all QDSCs, sub-bandgap photons absorption is observed in the range from $900 \mathrm{~nm}$ to $1100 \mathrm{~nm}$. All Si-doped samples display reduced the spectral response in the sub-bandgap region. This is primarily attributed to the extra electrons introduced by Si doping filling the $\mathrm{CB}$ of the QDs, which decreases the probability of the VB to IB transition. As a result, the absorption from the QDs is weakened [14]. Another possibility for the reduced EQE is the suppression of thermal escape of photo-excited carriers due to the formation of a potential barrier at $\mathrm{QD} / \mathrm{WL}$ interface by $\mathrm{Si}$ doping [4].

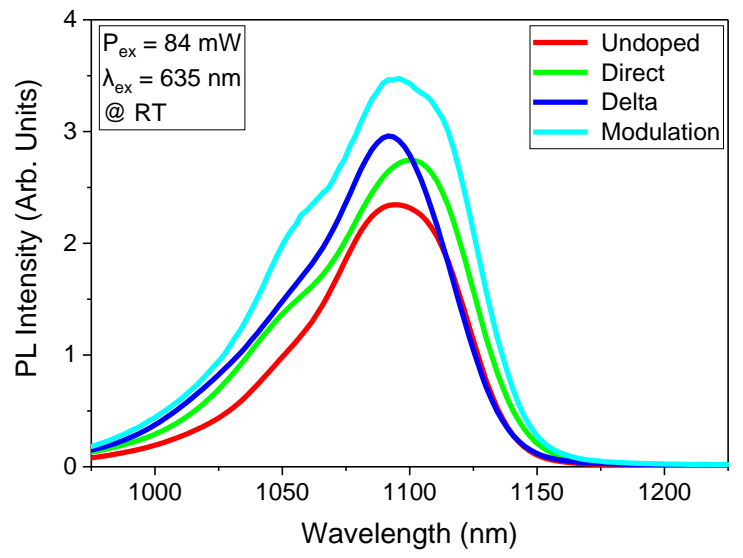

Figure 1 PL spectra of QDSC samples (R4 - R9). ( $\mathrm{P}_{\mathrm{ex}}=84 \mathrm{~mW}$ and $\lambda_{\mathrm{ex}}=635 \mathrm{~nm}$ at RT).

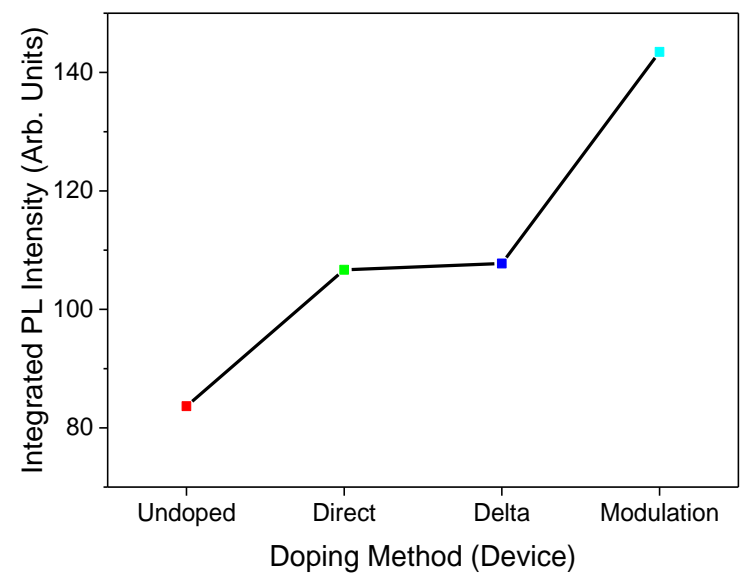

Figure 2 Integrated PL intensity vs. Si doping method at RT $\left(\mathrm{P}_{\mathrm{ex}}=84\right.$ $\left.\mathrm{mW}, \lambda_{\mathrm{ex}}=635 \mathrm{~nm}\right)$. 
The J-V characteristics of the all samples are presented in Figure 4. All doped QDSC samples have significantly lower current densities when compared with that of the undoped QDSC. This can be related to the reduction in the EQE contribution from the supra-bandgap region observed in Figure 3. In other words, the decrease in the depletion region width caused by the introduction of Si dopants results in a reduction in the effective area for absorption, and hence a decrease in the current density. All doped samples exhibit improvement in $\mathrm{V}_{\mathrm{OC}}$ compared to the undoped sample. This could be explained in terms of the reduced thermal coupling of QD states from the WL and CB in GaAs QDSCs assisted by $\mathrm{Si}$ doping. This reduced thermal coupling could be attributed to a potential barrier formed between the WL and QDs [4]. Delta and modulation-doped QDSCs display higher open-circuit voltages compared with the directly doped QDSC. This follows the same trend observed in the integrated PL intensity analysis, shown in Figure 2, which suggests that the reduced non-radiative recombination in the delta and modulationdoped QDSCs leads to the further voltage recovery.

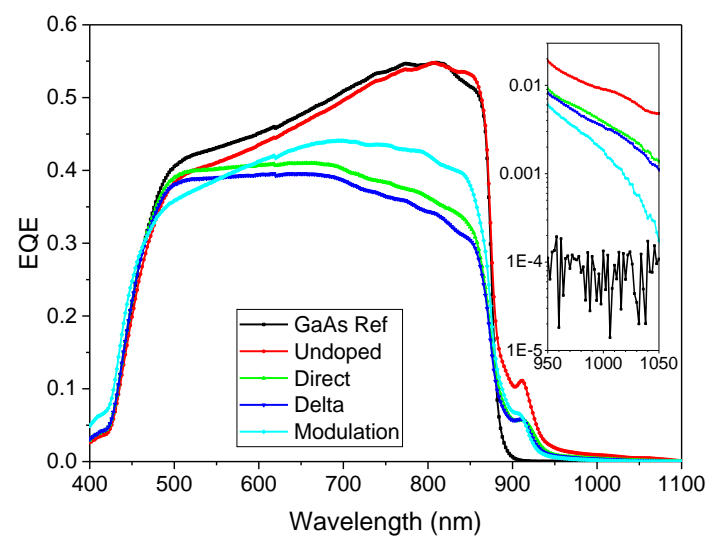

Figure 3 External quantum efficiency spectra for all SC samples.

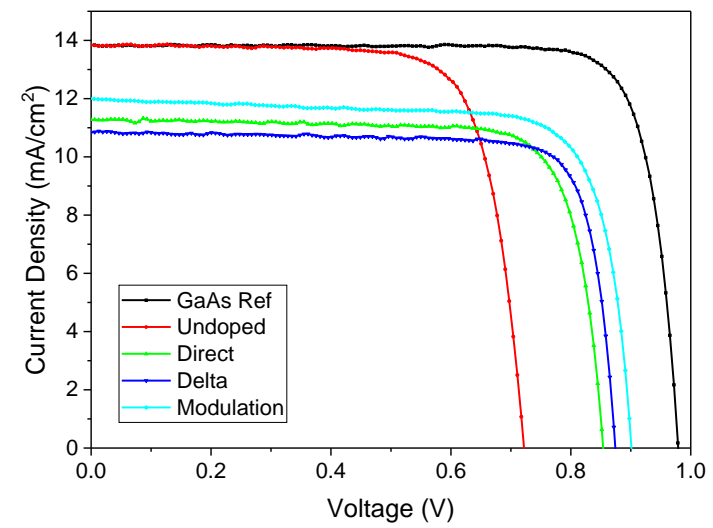

Figure 4 Current density vs voltage for all SC samples.

\section{CONCLUSION}

In conclusion, QDSCs with different doping methods and positions, alongside two reference cells, have been grown by MBE and characterised by PL, EQE, and J-V measurements. The results show a clear relationship between the degree of separation between the Si dopants and the QDs achieved by different doping methods, and the number of non-radiative recombination centres. All doped samples exhibited an increase in the $\mathrm{V}_{\mathrm{OC}}$ to the undoped QDSC. In particular, the modulation-doped QDSC shows improvements in both the current density and the voltage compared with the directly doped QDSC.

\section{REFERENCES}

[1] Y. Okada et al., 'Increase in photocurrent by optical transitions via intermediate quantum states in direct-doped InAs/GaNAs strain-compensated quantum dot solar cell', J. Appl. Phys., vol. 109, no. 2, p. 024301, Jan. 2011.

[2] F. K. Tutu et al., 'InAs/GaAs quantum dot solar cell with an AlAs cap layer', Appl. Phys. Lett., vol. 102, no. 16, p. 163907, Apr. 2013.

[3] I. Ramiro et al., 'InAs/AlGaAs quantum dot intermediate band solar cells with enlarged sub-bandgaps', 2012, pp. 000652 000656.

[4] P. Lam et al., 'Voltage recovery in charged InAs/GaAs quantum dot solar cells', Nano Energy, vol. 6, pp. 159-166, May 2014.

[5] S. J. Polly, D. V. Forbes, K. Driscoll, S. Hellstrom, and S. M. Hubbard, 'Delta-Doping Effects on Quantum-Dot Solar Cells', IEEE J. Photovolt., vol. 4, no. 4, pp. 1079-1085, Jul. 2014.

[6] K. A. Sablon, J. W. Little, V. Mitin, A. Sergeev, N. Vagidov, and K. Reinhardt, 'Strong Enhancement of Solar Cell Efficiency Due to Quantum Dots with Built-In Charge', Nano Lett., vol. 11, no. 6, pp. 2311-2317, 2011.

[7] E. Antolin et al., 'Advances in quantum dot intermediate band solar cells', in 2010 35th IEEE Photovoltaic Specialists Conference (PVSC), 2010, pp. 000065-000070.

[8] T. Morioka and Y. Okada, 'Dark current characteristics of InAs/GaNAs strain-compensated quantum dot solar cells', Phys. E Low-Dimens. Syst. Nanostructures, vol. 44, no. 2, pp. 390393, Nov. 2011.

[9] H. F. Lu, L. Fu, G. Jolley, H. H. Tan, and C. Jagadish, 'Improved performance of InGaAs/GaAs quantum dot solar cells using Si-modulation doping', in 2012 Conference on Optoelectronic and Microelectronic Materials Devices (COMMAD), 2012, pp. 127-128.

[10] F. K. Tutu et al., 'Improved performance of multilayer InAs/GaAs quantum-dot solar cells using a high-growthtemperature GaAs spacer layer', J. Appl. Phys., vol. 111, no. 4, p. 046101, Feb. 2012.

[11] H. Y. Liu et al., 'Improved performance of $1.3 \mu \mathrm{m}$ multilayer InAs quantum-dot lasers using a high-growth-temperature GaAs spacer layer', Appl. Phys. Lett., vol. 85, no. 5, pp. 704-706, Aug. 2004.

[12] H. Y. Liu et al., 'p-doped $1.3 \mu \mathrm{mInAs} / \mathrm{GaAs}$ quantum-dot laser with a low threshold current density and high differential efficiency', Appl. Phys. Lett., vol. 89, no. 7, p. 073113, Aug. 2006. 
[13] H. Y. Liu et al., 'Influences of the spacer layer growth temperature on multilayer InAs/GaAs quantum dot structures', J. Appl. Phys., vol. 96, no. 4, pp. 1988-1992, Aug. 2004.

[14] X. Yang et al., 'Improved efficiency of InAs/GaAs quantum dots solar cells by Si-doping', Sol. Energy Mater. Sol. Cells, vol. 113, pp. 144-147, Jun. 2013.
[15] X. Lu, J. Vaillancourt, and M. J. Meisner, 'A modulation-doped longwave infrared quantum dot photodetector with high photoresponsivity', Semicond. Sci. Technol., vol. 22, no. 9, p. 993, 2007. 